\title{
3D Printing and Weibull statistical evaluations of nanographene-containing ABS thermoplastics
}

Robert Bubeck ${ }^{1}$, Stephen Gillman ${ }^{2}$, Peter Kozerski ${ }^{3}$, Michael Most ${ }^{4}$, Tracy Zhang ${ }^{1}$

${ }^{1}$ St. Andrews Campus, Michigan State University, Midland, MI

2 Michigan Technological University, MI

${ }^{3}$ H. H. Dow High School, Midland, MI

${ }^{3}$ Michigan State University, East Lansing, MI

ACS-PMSE 3286812. To have been presented at 2:35 PM, March 22, 2020 bubeck@msu.edu 


\section{Material parameters (1):}

\section{ABS - Trinseo Magnum ${ }^{\text {TM }} 347 E Z$}

- Styrene* 75.3 wt. \%

- Acrylonitrile* $16.4 \mathrm{wt}$.

- Butadiene **

8.3 wt. \%

- $\mathrm{M}_{\mathrm{w}}=160,600$

- TGA: - 0.5 Wt.\%, 15 min., $240^{\circ} \mathrm{C}$ in air

${ }^{*}{ }^{13}$ C NMR

$* *{ }^{1} \mathrm{H}$ NMR

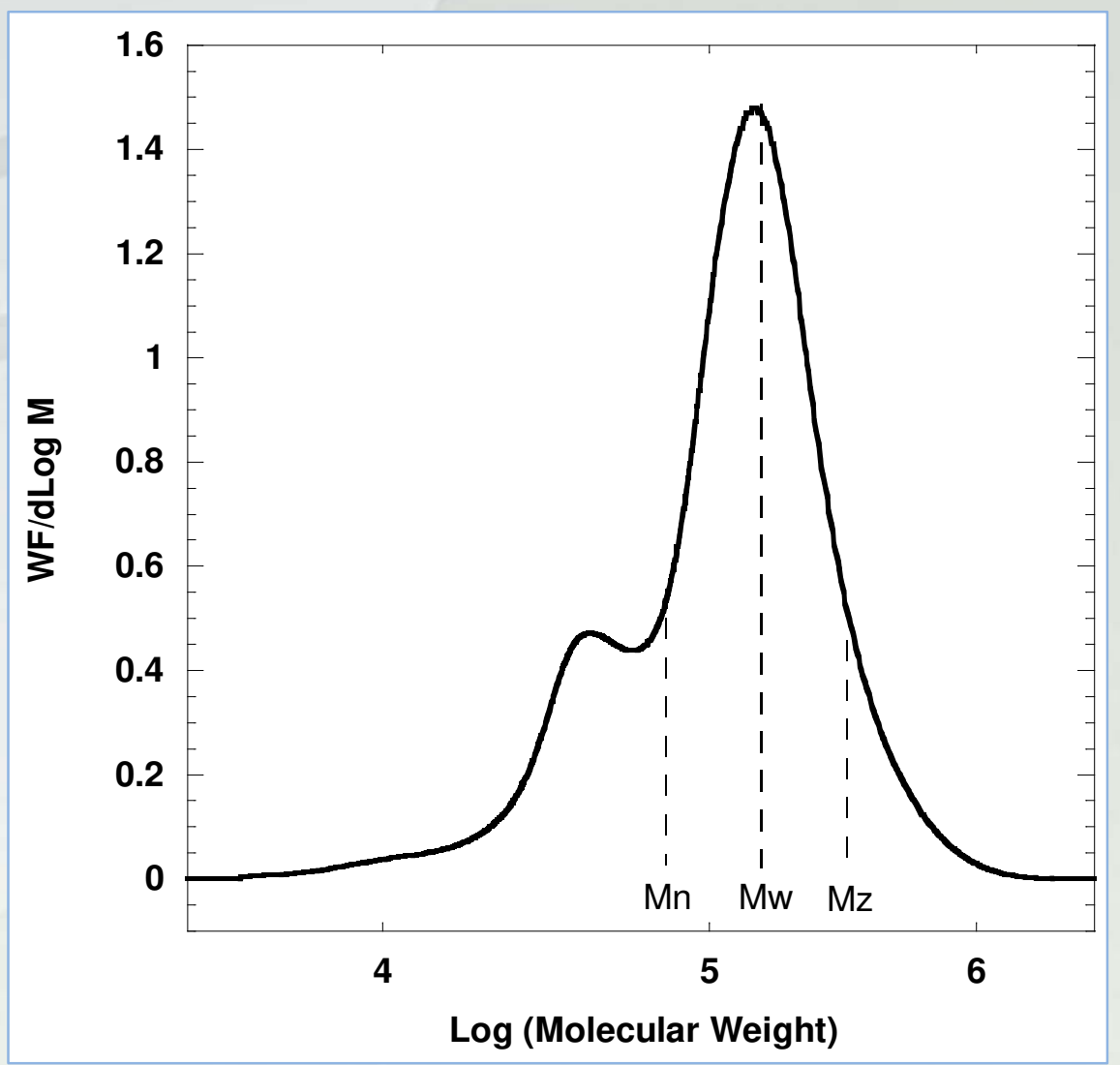




\section{Material parameters (2):}

+ XGSciences XGnP ${ }^{\mathrm{TM}}$ M5 Graphene

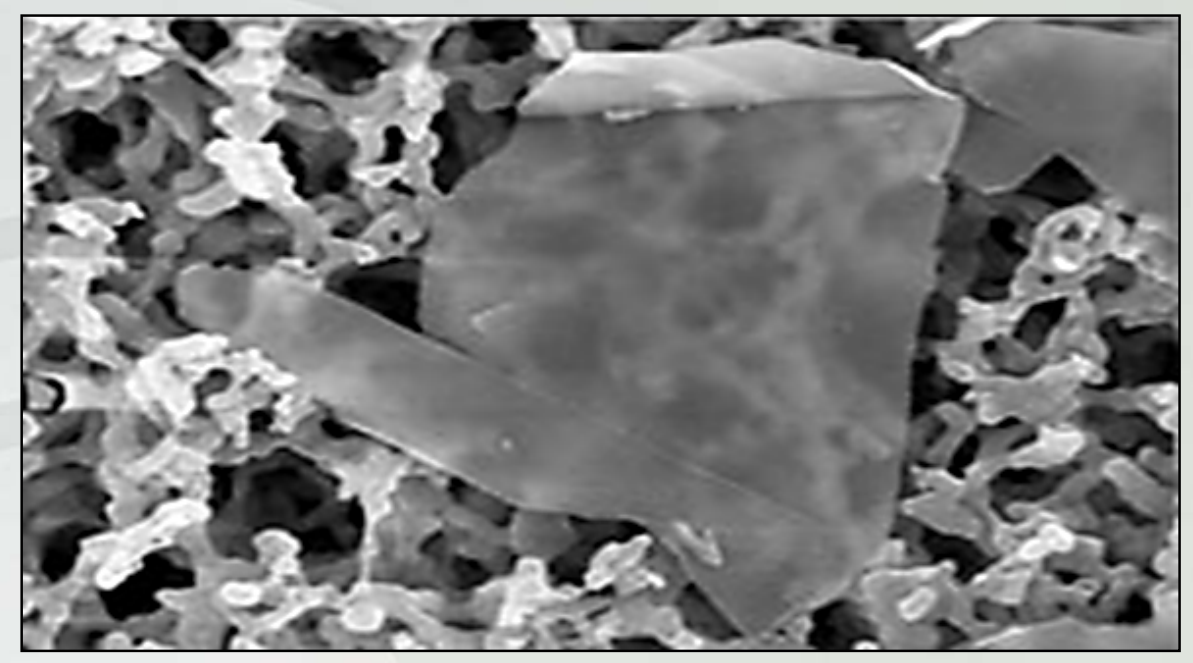

- M5: $5 \mu$ dia., 5-10 $\mathrm{nm}$ thick, $150 \mathrm{~m}^{2} / \mathrm{g}$ surface area, $\mathrm{E}$ (in-plane) $=1.0 \mathrm{TPa}$.

- Compounded using a twin-screw extruder to levels of $\mathbf{0 , 5}$, and 10 wt.\%.

- Material Extrusion (MatEx) Fabrication -- 3D printing using FlashForge Dreamer and Fusion 3 F410 3D printers.

$\checkmark$ See related example: S. F. A. Acquah et al. "Carbon Nano-tubes and Graphene as Additives in 3D Printing" in Carbon nanotubes Current Progress of Their Polymer Composites, Ch. 8, 227 (2016) Intech. 


\section{Compounding and filament fabrication:}

- Magnum ${ }^{\mathrm{TM}}$ 347EZ ABS compounded with XGSciences XGnP ${ }^{\mathrm{TM}}$ M5 graphene by Midland Compounding and Consulting.

- Werner-Pfleiderer co-rotating, twin screw extruder: Zone temperatures: $200^{\circ} \mathrm{C}$ to $220^{\circ} \mathrm{C}$.

- Compounded material was cast from a strand die onto a chilled stainless-steel belt and pelletized.

- Filament fabricated by 3DXTECH, Grand Rapids, MI.

$>$ Dia. $=1.76 \mathrm{~mm}+/-1.5$. 


\section{EZ ABS w/10 wt.\% M5 - Edge vs. Center}
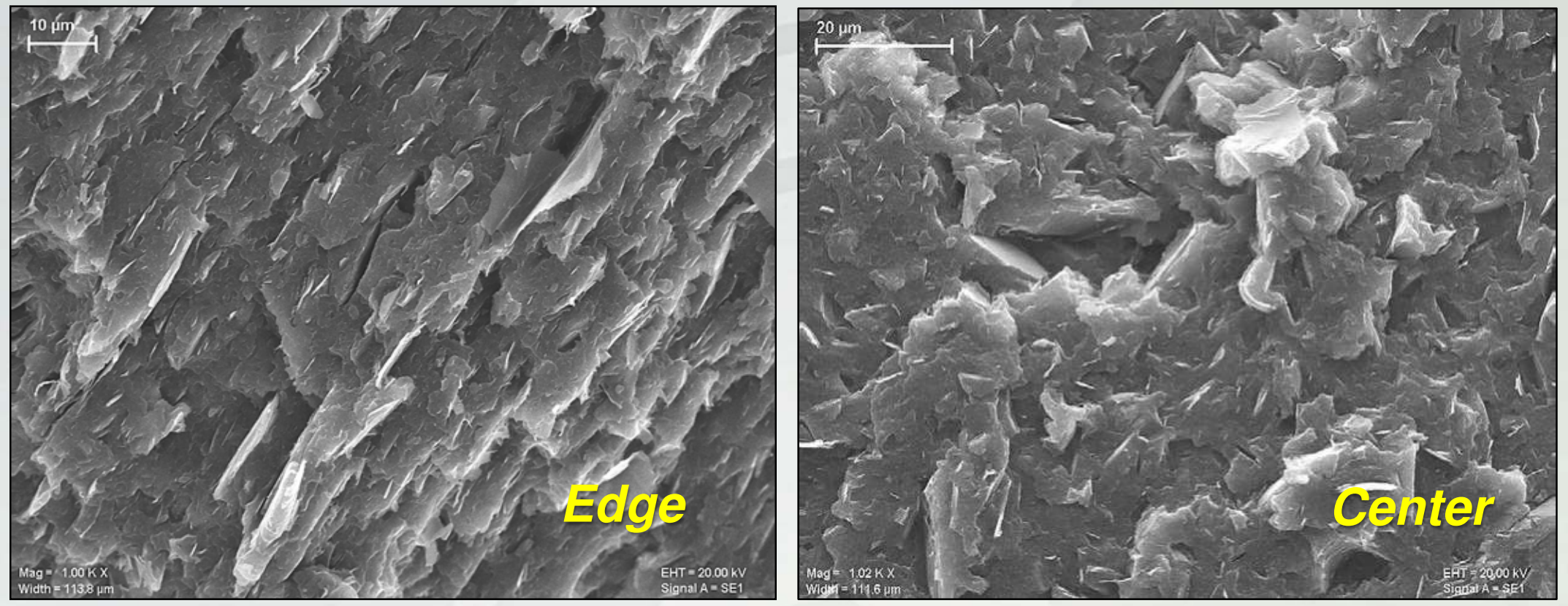

> M5 Platelets circumferentially oriented in the outer $100 \mu \mathrm{m}$ but randomly oriented in the filament center. 


\section{$347 E Z$ ABS w/5\% M5 (45-45 XY raster angle example):}
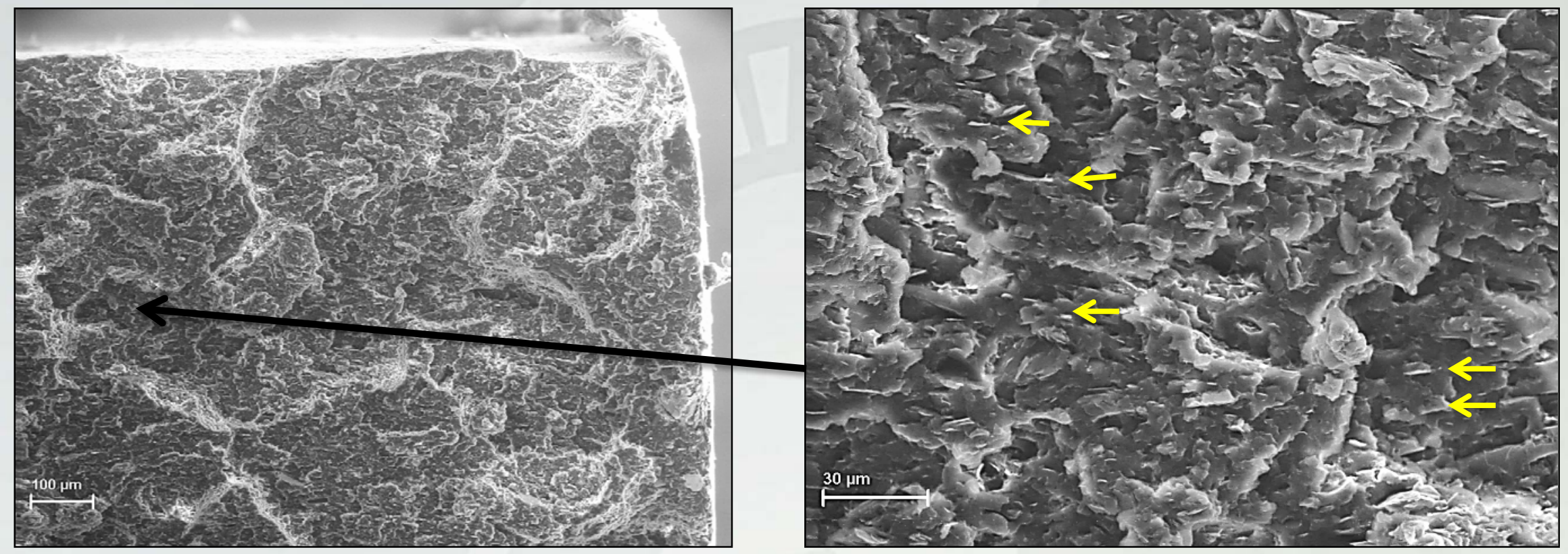

$>$ Graphene platelets tend to align with print bed plane 


\section{FlashForge Dreamer}

$>$ Dual direct extruders in print head

$>$ Manual bed leveling

$>3 \mathrm{M}$ print platform

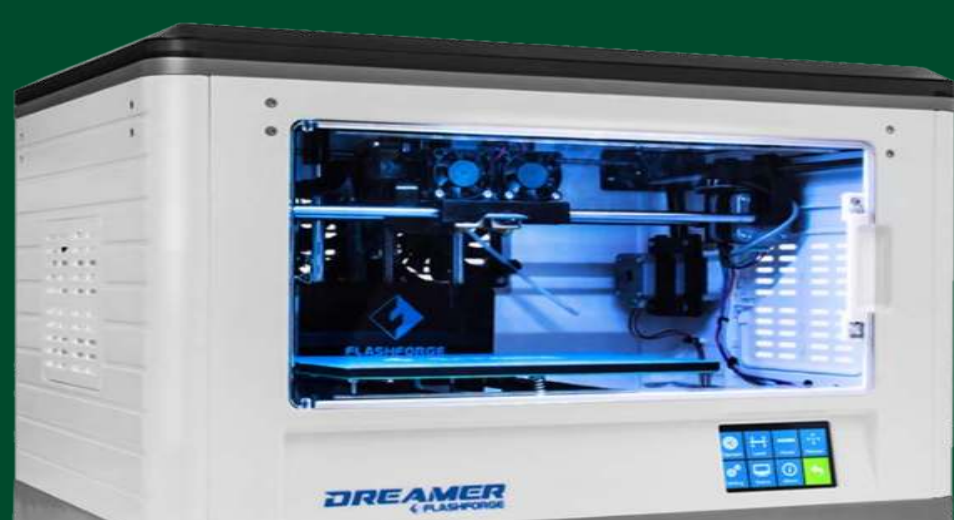

\section{Fusion 3 F410}

$>$ Single Bowden tube extruder

> Higher print temperatures and a larger area

$>$ Faster print speeds

$>$ Automatic glass bed leveling

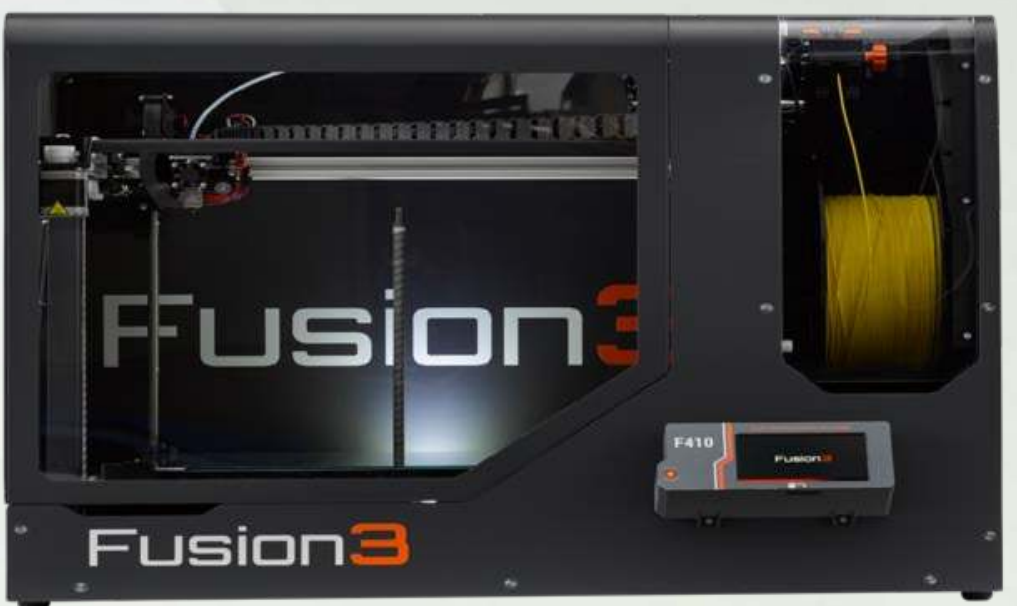




\section{FlashForge - Tensile Elongation:}

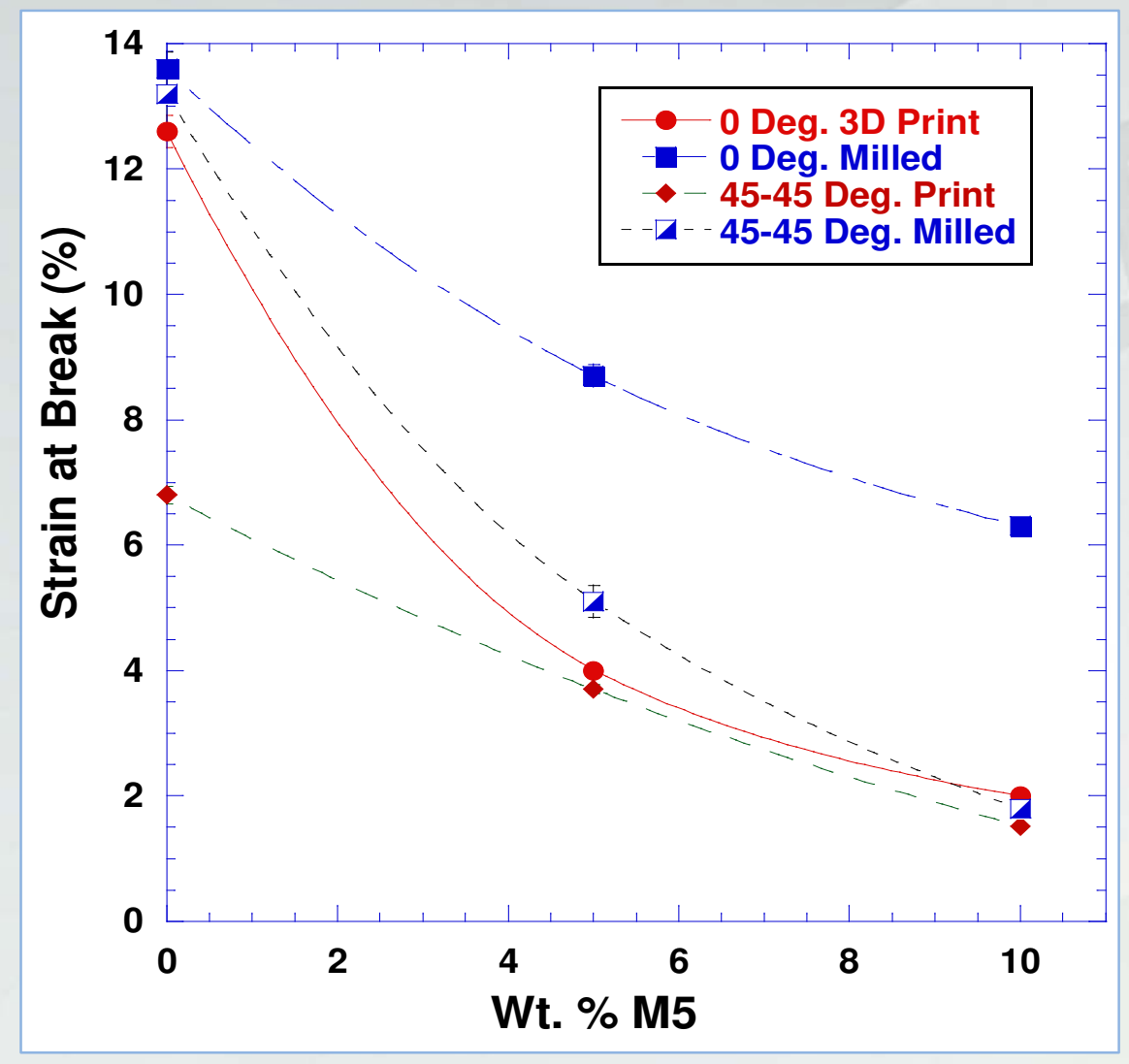

- All tensile data @ 0.2 in $/ \mathrm{min}$

- ASTM 638D (2010)

- NISTIR 859 compliant

See: Bubeck et al. in Seppala et al.; Polymer-Based Additive Manufacturing: Recent Developments, ACS Symposium Series; American Chemical Society: Washington, DC, (2019). 
FlashForge: -Tensile Modulus:

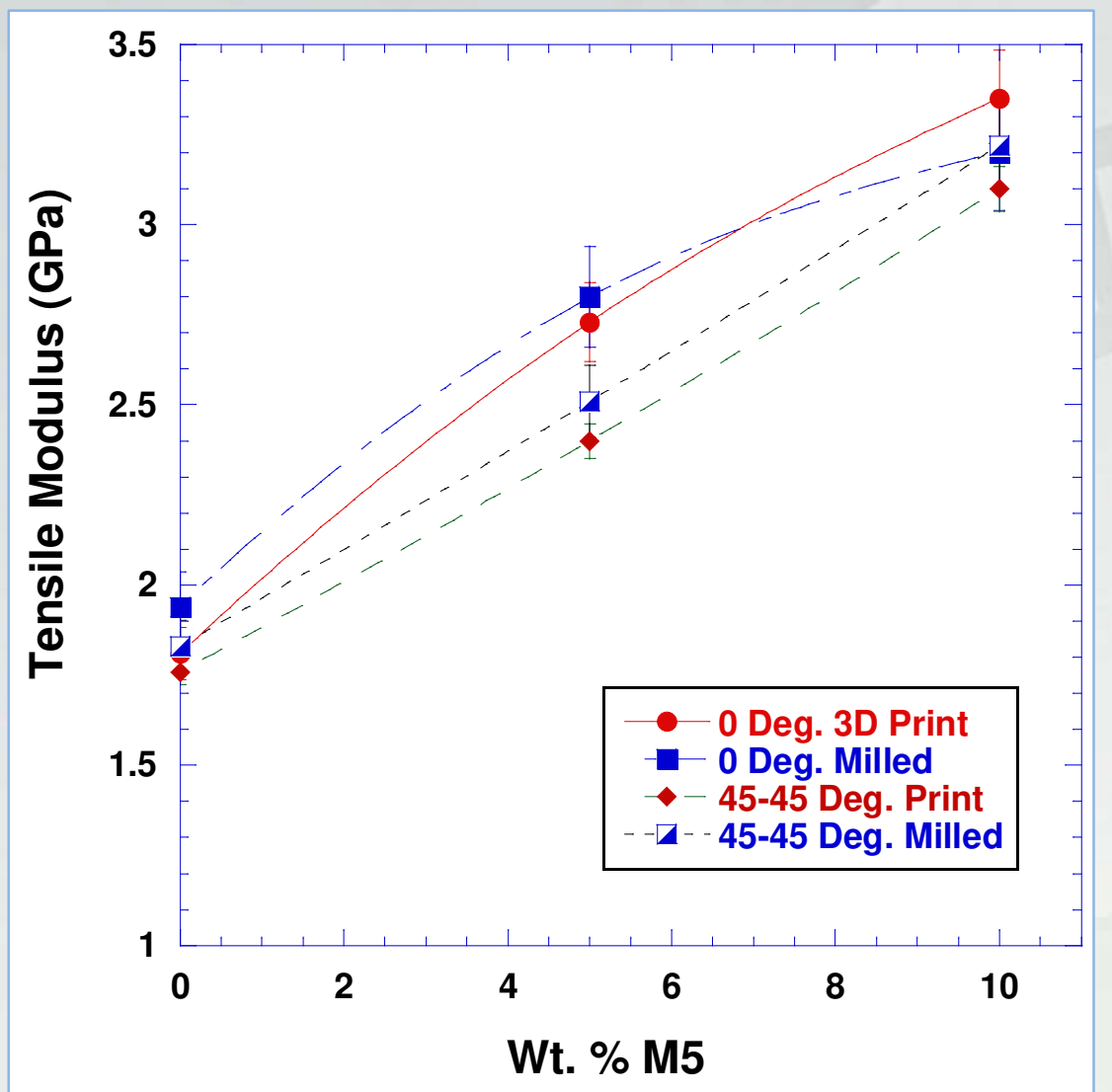

-Tensile Strength:

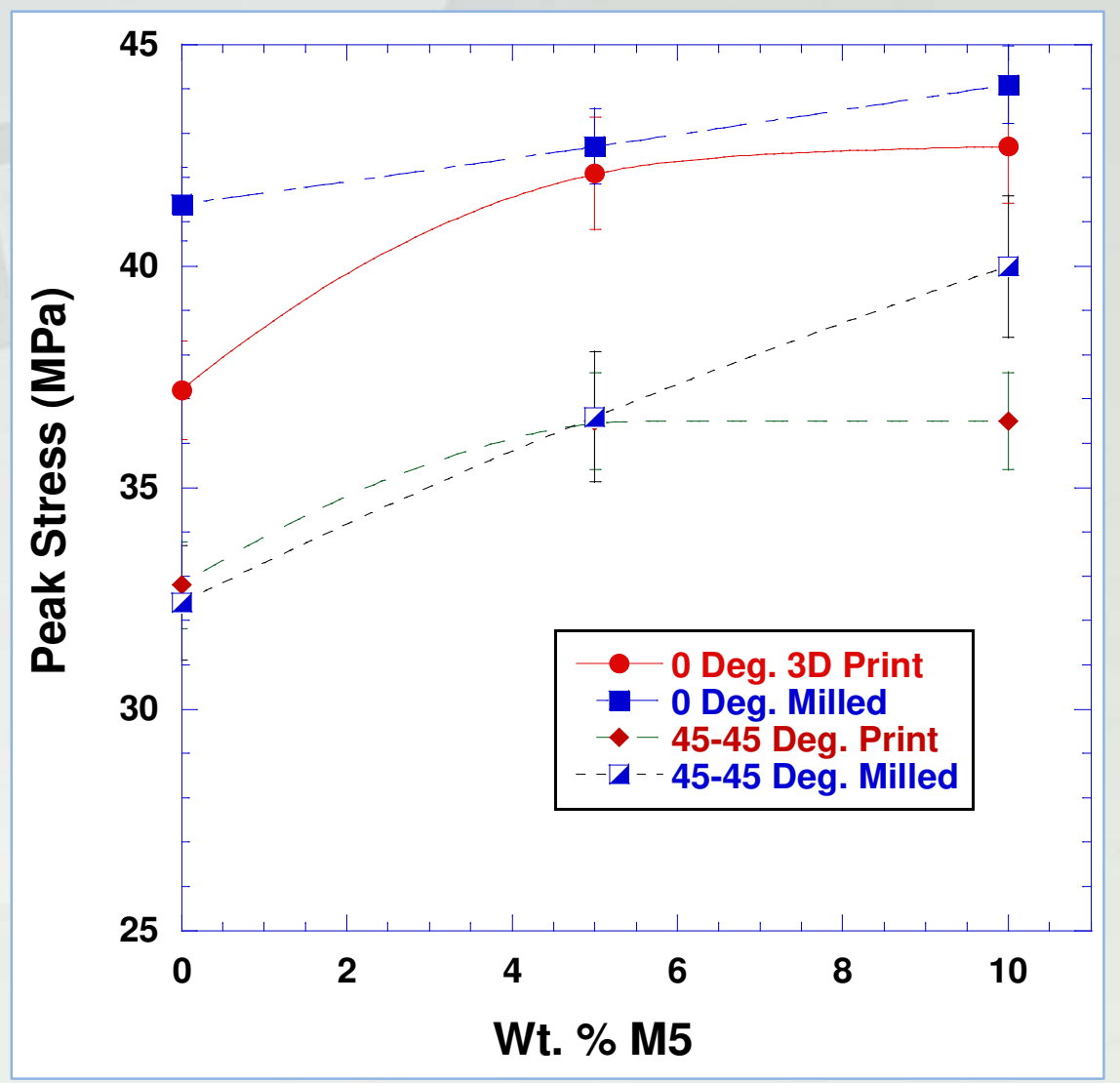


Circumferential (1) and internal (2) 3D pattern for a 45-45 deg. raster angle tensile bar:

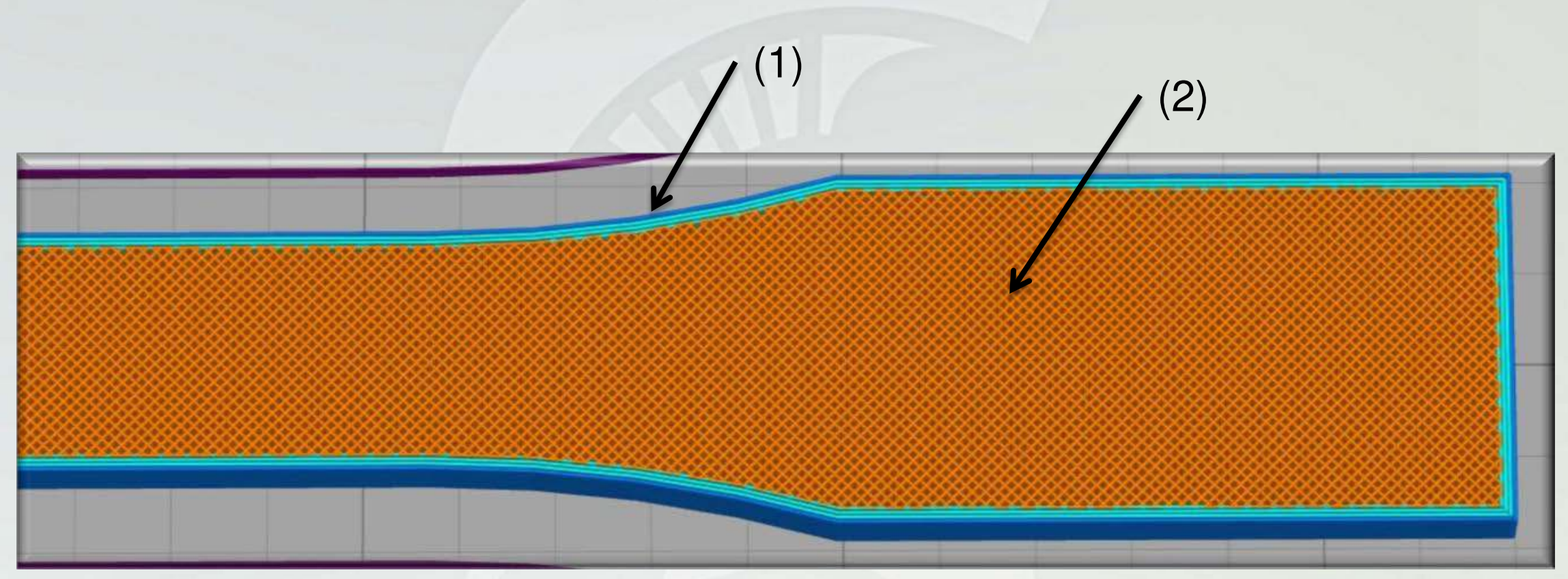




\section{Example optical micrographs of fracture surfaces (FlashForge):}

Delamination of surface layers associated with circumferential MatEx printing

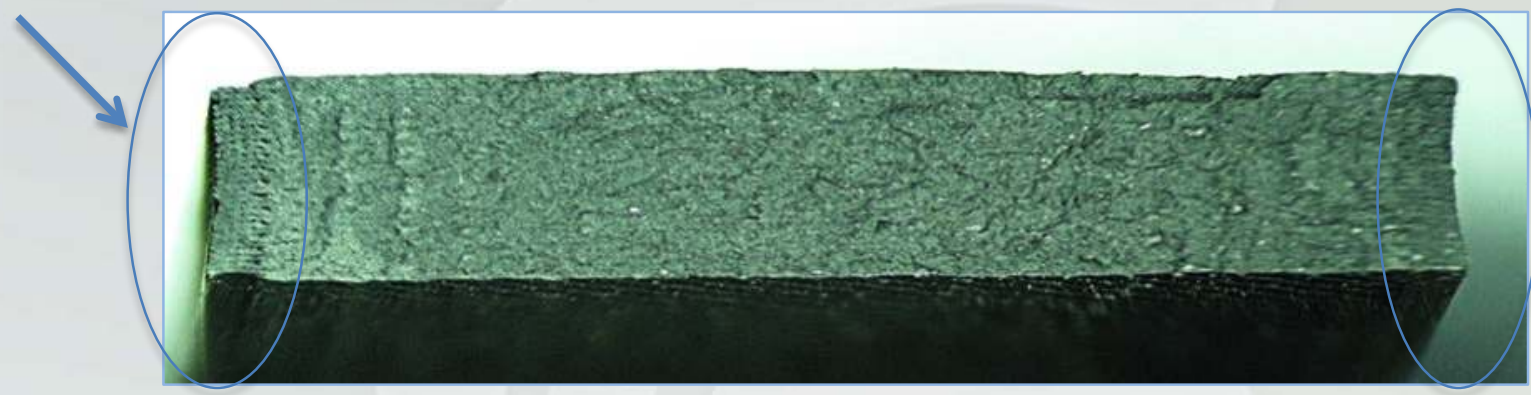

Print-to-shape ABS $45^{\circ}-45^{\circ} 5$ wt.\% M5. Elongation: 3.7\%

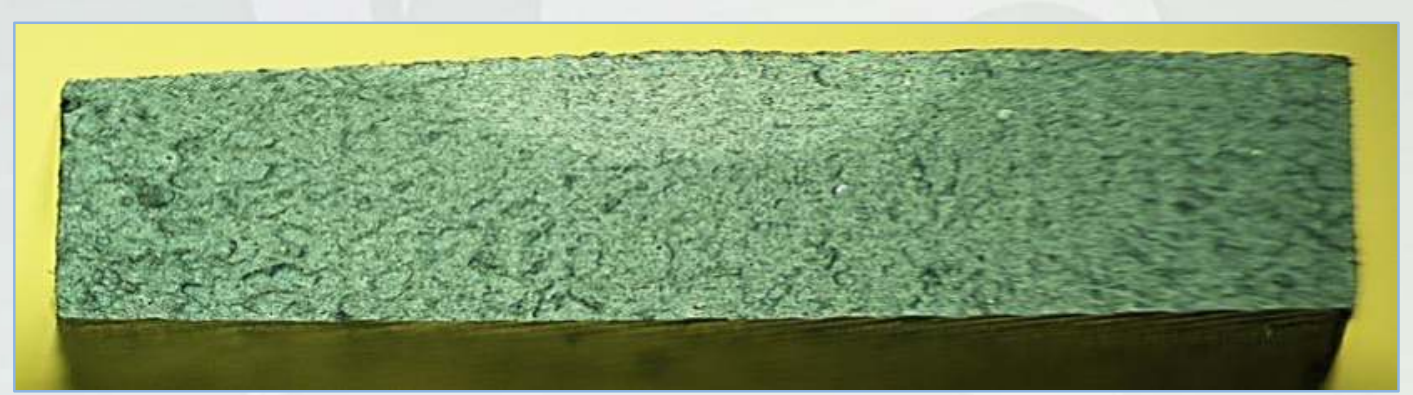

Note:

Internal filament bead fusion is very good!

Milled-to-shape ABS $45^{\circ}-45^{\circ} 5$ wt.\% M5. Elongation: 5.1\% 


\section{Comparison w/Epon-m-PDA/M5 Nanocomposite:}

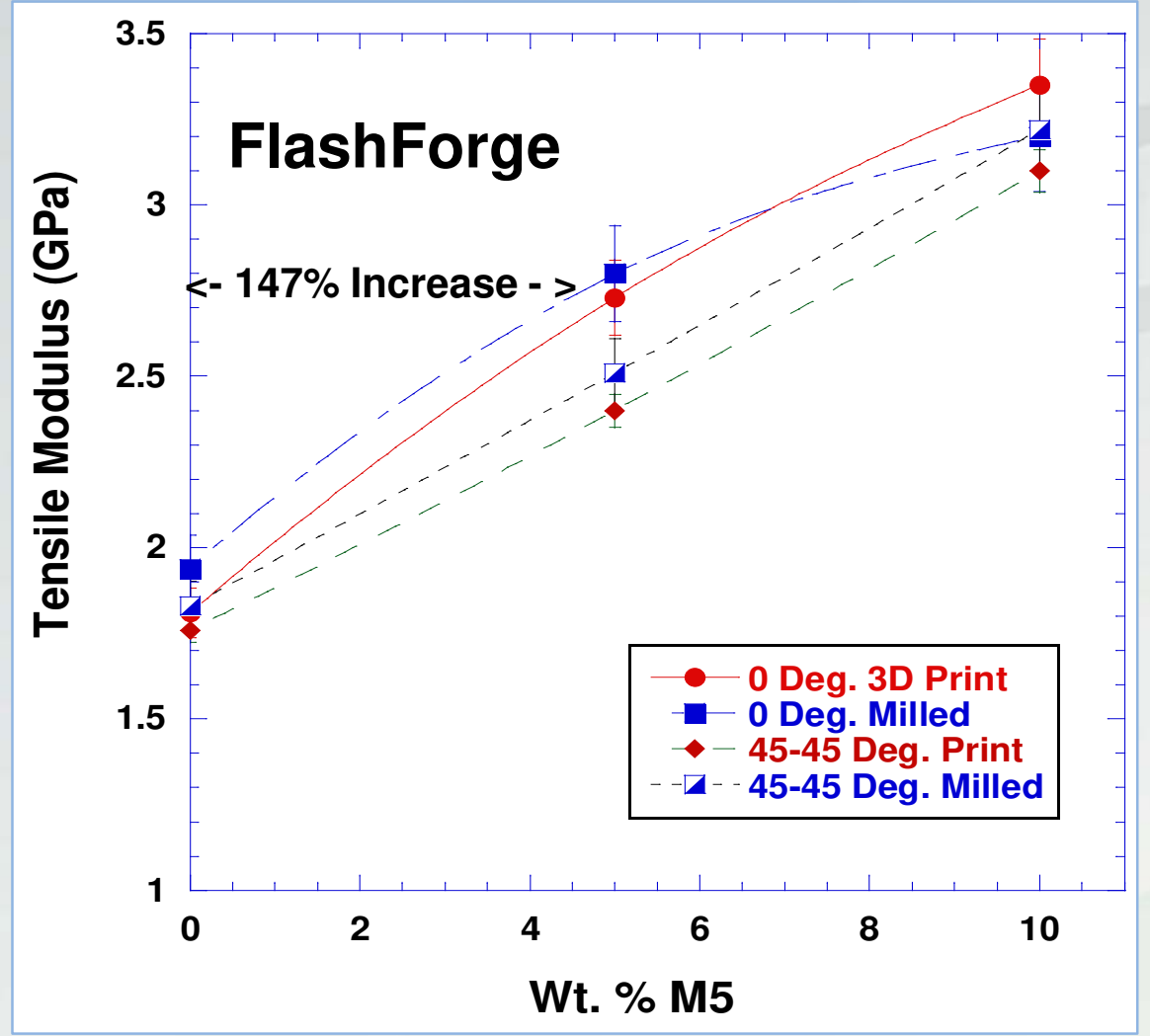

J Mater Sci (2015) 50:1082-1093 *

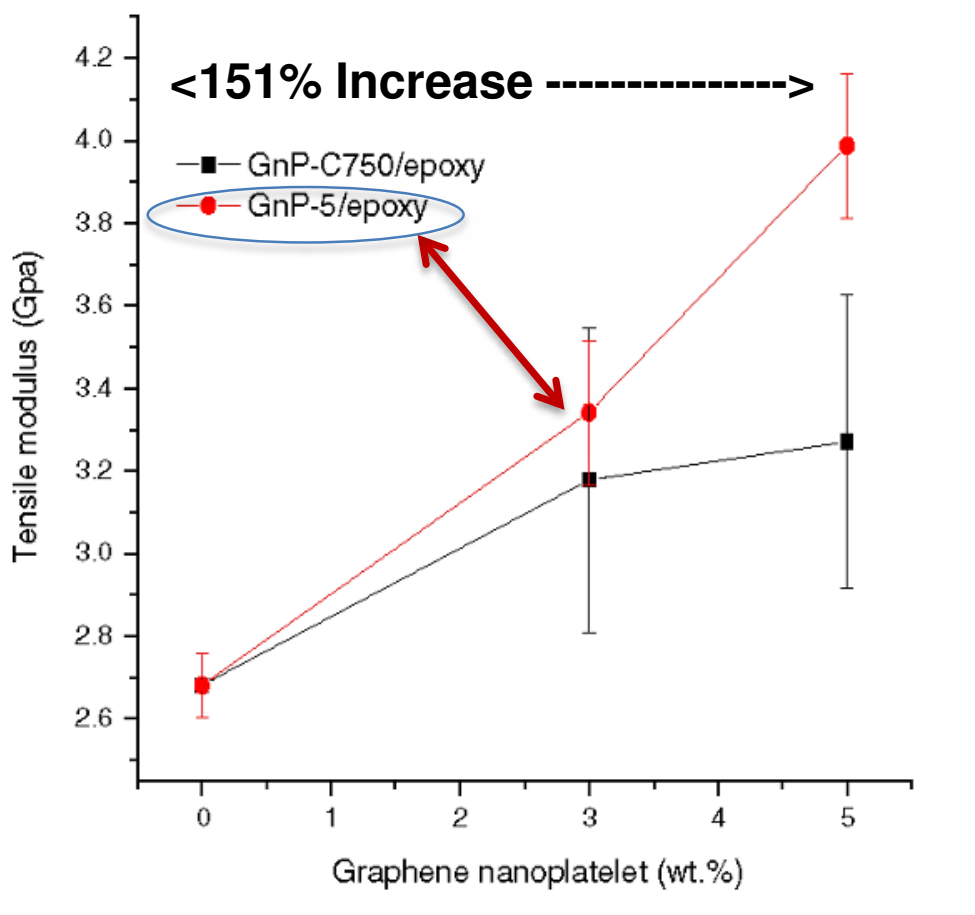

${ }^{*}$ F. Wang et al. J. Mater. Sci. (2015) 50:1082-1093 


\section{Print-to-Shape - Young's Modulus: FlashForge vs. Fusion:}
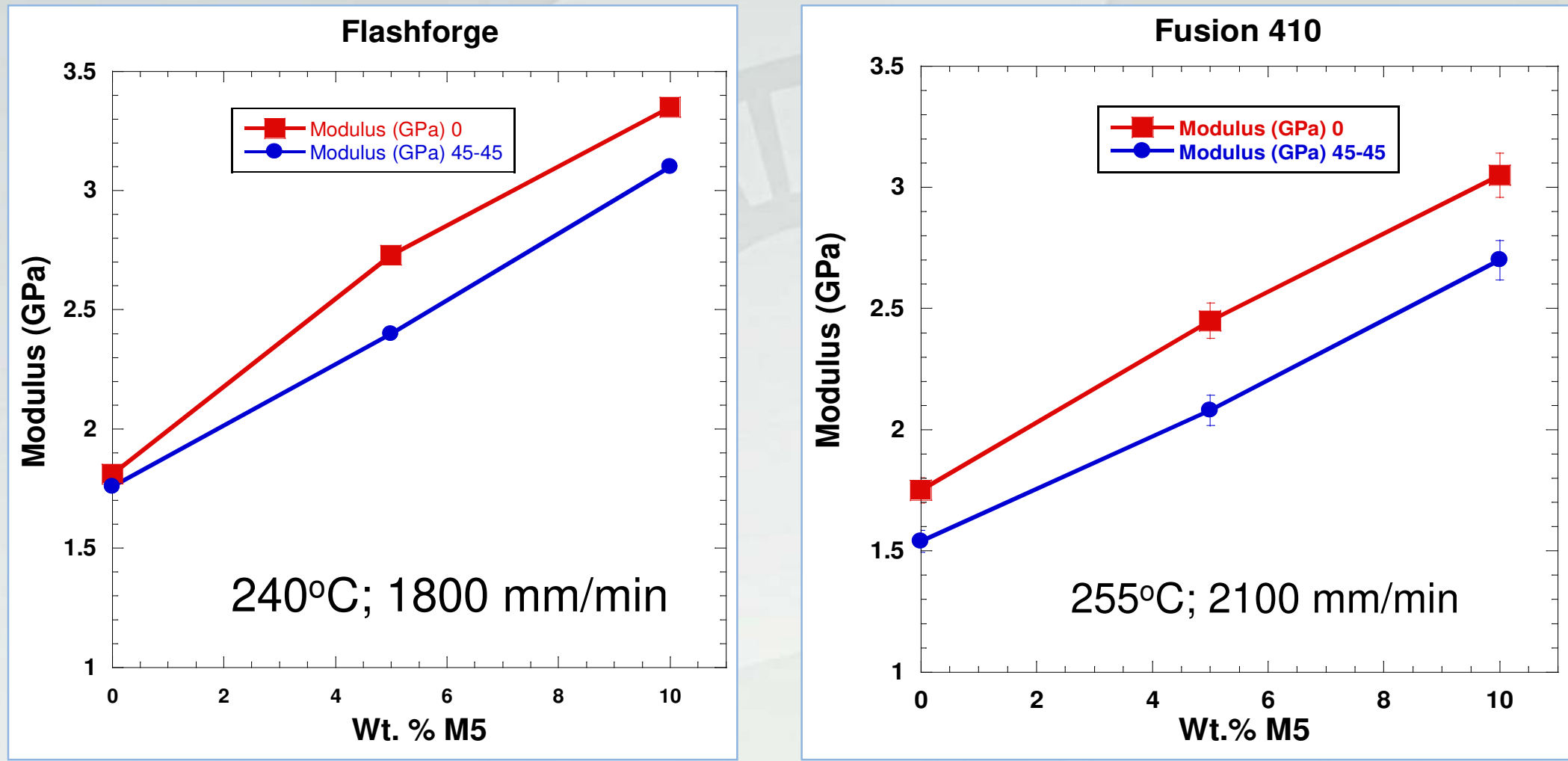


\section{Weibull analysis tensile sample characteristics:}

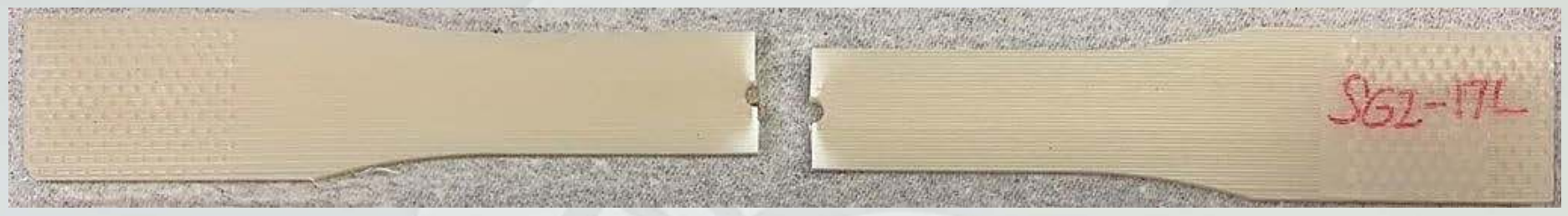

- 3D printed samples milled-to-shape according to ASTM D638-10.

- $3 \mathrm{~mm}$ diameter holes were printed in place in middle of sample.

- ABS samples were fabricated using both printers.

- PLA samples were fabricated using the FlashForge only.

- For further reading, see:

$\checkmark$ W. Weibull, J. Appl. Mech., (1953) 18, 3, 293-297.

$\checkmark$ O. Keles et al., Rapid Prototyping Journal, (2017) 23/2, 320-328. 


\section{Weibull Analysis:}

Two-parameter Weibull distribution to relate probability of failure and applied stress (volume as the second parameter is omitted by assuming it equal to one due to very low strain):

$$
F(\sigma)=1-\exp \left[-\left(\sigma / \sigma_{o}\right)^{m}\right]
$$

Weibull plots are generated by sorting raw data (yield stresses) in ascending order, assigning ranks to these data and calculating $F(\sigma)$ with:

$$
\text { F with } F=(1-0.5) / N \text {. }
$$

A Weibull plot is constructed from $\ln (\ln (1 /(1-F)))$ vs. $\ln (\sigma)$. The linear fit equation from the plot is manipulated to construct a failure probability graph based on a set failure rate $(F)$ for its corresponding stress estimate. 


\section{Weibull Plot Examples:}
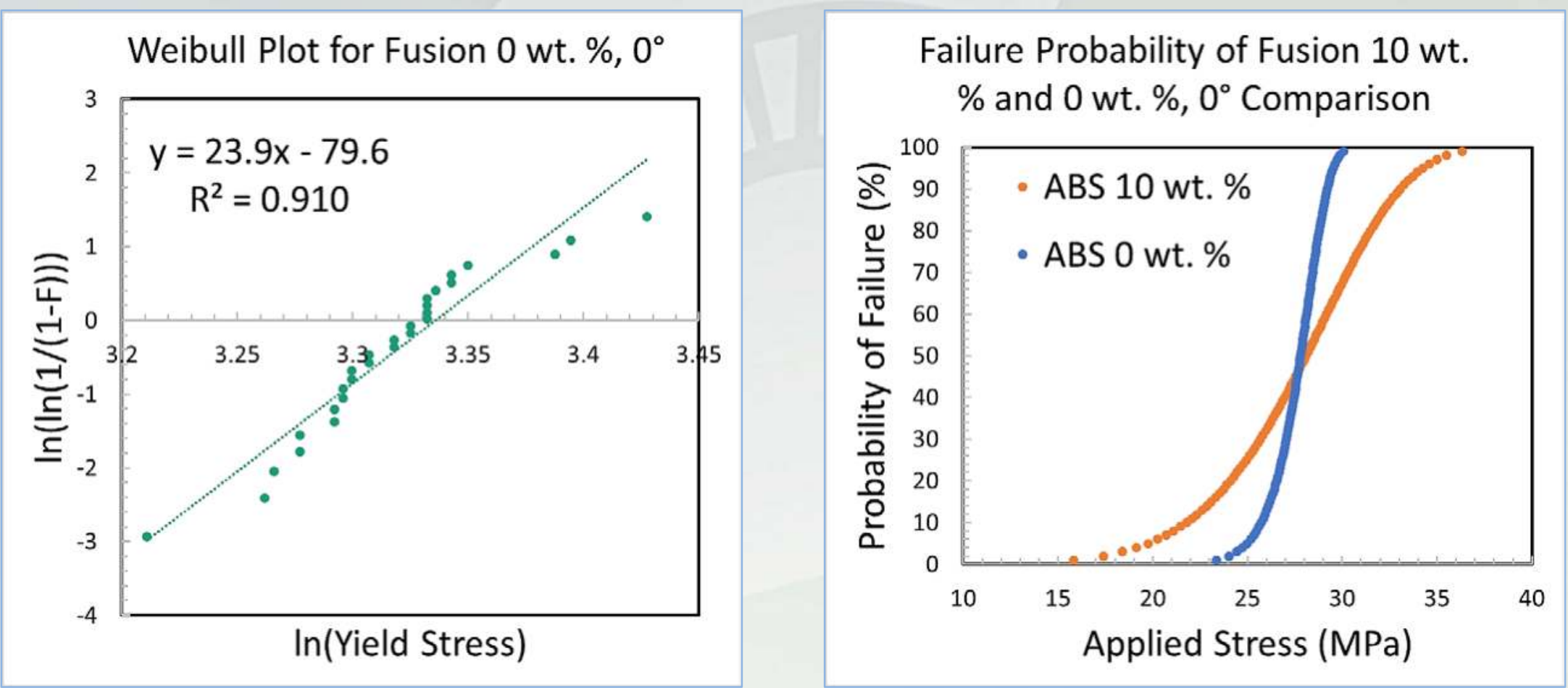


\section{Weibull Results:}

\begin{tabular}{|c|c|c|c|c|c|c|c|}
\hline Printer & $\begin{array}{c}\text { Material } \\
\text { (wt. \% M5) }\end{array}$ & $\begin{array}{l}\text { Raster } \\
\text { Angle } \\
\text { (deg.) }\end{array}$ & $\begin{array}{c}\text { Hole } \\
(3 \mathrm{~mm})\end{array}$ & Weibull Fit & $\begin{array}{c}\text { Mean Yield } \\
\text { Stress } \\
\text { (MPa) }\end{array}$ & $\begin{array}{l}\text { Mean } \\
\text { Modulus } \\
\text { (GPa) } \\
+/-5 \%\end{array}$ & $\begin{array}{c}\text { Mean } \\
\text { Strain at } \\
\text { Break (\%) }\end{array}$ \\
\hline \multirow{4}{*}{ Fusion (F) } & ABS & 0 & \multirow{7}{*}{ Yes } & $y=23.9 x-80$ & $28+/-1$ & 1.78 & 1.8 \\
\hline & ABS 5\% & 0 & & $y=24.0 x-80$ & $29+/-1$ & 2.48 & 1.5 \\
\hline & $A B S 10 \%$ & 0 & & $y=7.38 x-25$ & $28+/-2$ & 3.28 & 1.0 \\
\hline & ABS $10 \%$ & $45-45$ & & $y=23.0 x-74$ & $24+/-1$ & 2.72 & 1.1 \\
\hline \multirow{4}{*}{$\begin{array}{c}\text { FlashForg } \\
\text { e (FF) }\end{array}$} & ABS & 0 & & $y=26.7 x-87$ & $26+/-1$ & 1.82 & 1.7 \\
\hline & $A B S 10 \%$ & 0 & & $y=6.83 x-22$ & $23+/-2$ & 2.93 & 1.0 \\
\hline & PLA & 0 & & $y=47 x-179$ & $45+/-1$ & 2.89 & 1.9 \\
\hline & PLA & 0 & No & $y=39 x-159$ & $61+/-2$ & 2.85 & 2.7 \\
\hline
\end{tabular}




\section{Weibull Results:}

\begin{tabular}{|c|c|c|c|c|c|c|c|}
\hline Printer & $\begin{array}{c}\text { Material } \\
\text { (wt. \% M5) }\end{array}$ & $\begin{array}{l}\text { Raster } \\
\text { Angle } \\
\text { (deg.) }\end{array}$ & $\begin{array}{c}\text { Hole } \\
(3 \mathrm{~mm})\end{array}$ & Weibull Fit & $\begin{array}{c}\text { Mean Yield } \\
\text { Stress } \\
\text { (MPa) }\end{array}$ & $\begin{array}{l}\text { Mean } \\
\text { Modulus } \\
\text { (GPa) } \\
+/-5 \%\end{array}$ & $\begin{array}{c}\text { Mean } \\
\text { Strain at } \\
\text { Break (\%) }\end{array}$ \\
\hline \multirow{4}{*}{ Fusion (F) } & $A B S$ & 0 & \multirow{7}{*}{ Yes } & $y=23.9 x-80$ & $28+/-1$ & 1.78 & 1.8 \\
\hline & $A B S 5 \%$ & 0 & & $y=24.0 x-80$ & $29+/-1$ & 2.48 & 1.5 \\
\hline & ABS $10 \%$ & 0 & & $y=7.38 x-25$ & $28+/-2$ & 3.28 & 1.0 \\
\hline & $A B S 10 \%$ & $45-45$ & & $y=23.0 x-74$ & $24+/-1$ & 2.72 & 1.1 \\
\hline \multirow{4}{*}{$\begin{array}{c}\text { FlashForge } \\
\text { (FF) }\end{array}$} & $A B S$ & 0 & & $y=26.7 x-87$ & $26+/-1$ & 1.82 & 1.7 \\
\hline & ABS $10 \%$ & 0 & & $y=6.83 x-22$ & $23+/-2$ & 2.93 & 1.0 \\
\hline & PLA & 0 & & $y=47 x-179$ & $45+/-1$ & 2.89 & 1.9 \\
\hline & PLA & 0 & No & $y=39 x-159$ & $61+/-2$ & 2.85 & 2.7 \\
\hline
\end{tabular}




\section{Photomicrographs of ABS Weibull Fracture Surfaces:}

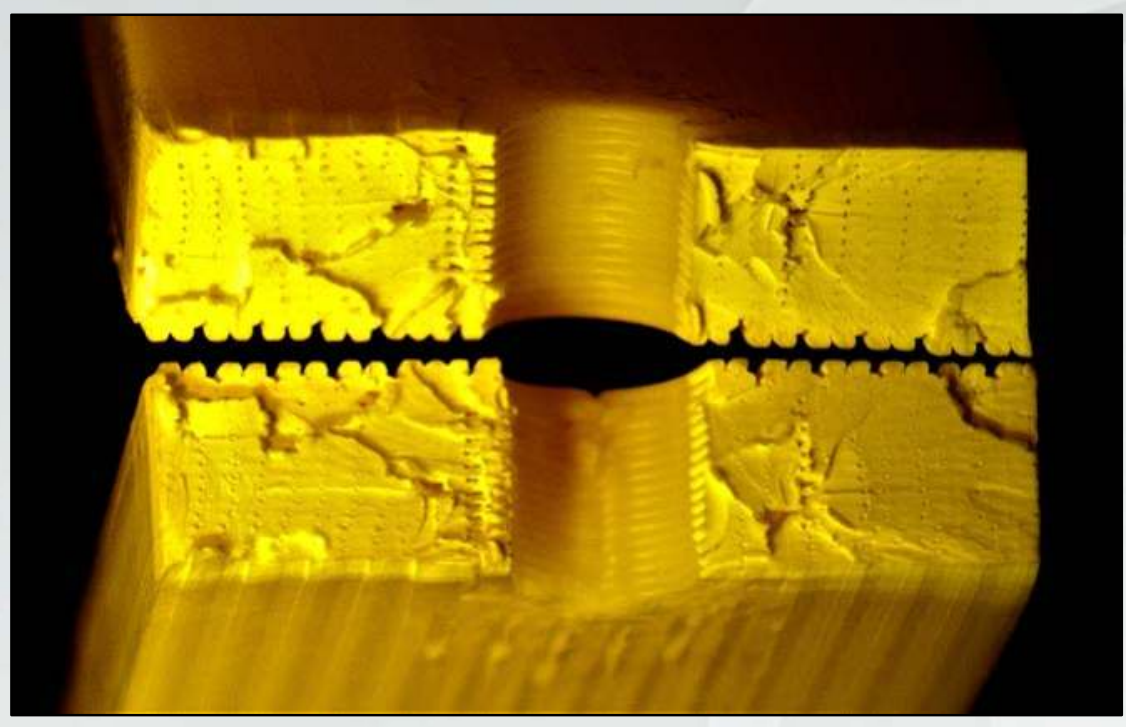

ABS

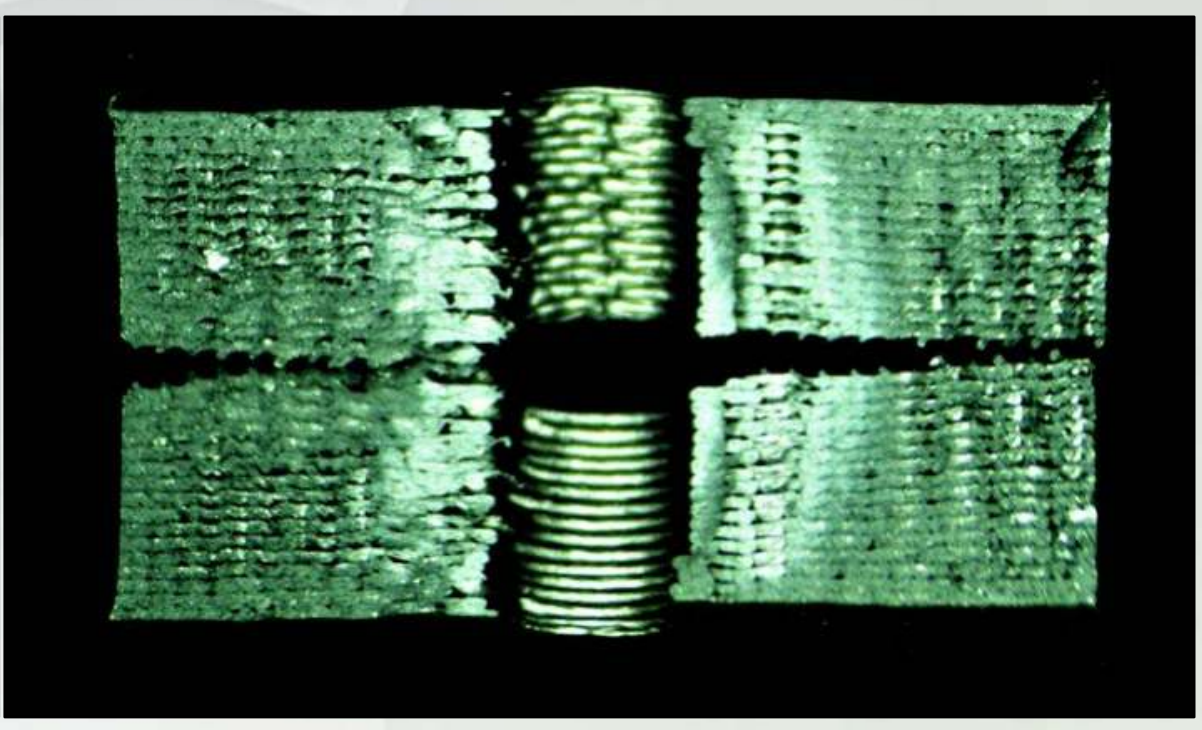

ABS/10 wt. \% M5 


\section{Weibull Results:}

\begin{tabular}{|c|c|c|c|c|c|c|c|}
\hline Printer & $\begin{array}{c}\text { Material } \\
\text { (wt. \% M5) }\end{array}$ & $\begin{array}{l}\text { Raster } \\
\text { Angle } \\
\text { (deg.) }\end{array}$ & $\begin{array}{c}\text { Hole } \\
(3 \mathrm{~mm})\end{array}$ & Weibull Fit & $\begin{array}{c}\text { Mean Yield } \\
\text { Stress } \\
\text { (MPa) }\end{array}$ & $\begin{array}{l}\text { Mean } \\
\text { Modulus } \\
\text { (GPa) } \\
+/-5 \%\end{array}$ & $\begin{array}{c}\text { Mean } \\
\text { Strain at } \\
\text { Break (\%) }\end{array}$ \\
\hline \multirow{4}{*}{ Fusion (F) } & $A B S$ & 0 & \multirow{7}{*}{ Yes } & $y=23.9 x-80$ & $28+/-1$ & 1.78 & 1.8 \\
\hline & ABS 5\% & 0 & & $y=24.0 x-80$ & $29+/-1$ & 2.48 & 1.5 \\
\hline & ABS $10 \%$ & 0 & & $y=7.38 x-25$ & $28+/-2$ & 3.28 & 1.0 \\
\hline & ABS $10 \%$ & $45-45$ & & $y=23.0 x-74$ & $24+/-1$ & 2.72 & 1.1 \\
\hline \multirow{4}{*}{$\begin{array}{c}\text { FlashForge } \\
\text { (FF) }\end{array}$} & $A B S$ & 0 & & $y=26.7 x-87$ & $26+/-1$ & 1.82 & 1.7 \\
\hline & ABS $10 \%$ & 0 & & $y=6.83 x-22$ & $23+/-2$ & 2.93 & 1.0 \\
\hline & PLA & 0 & & $y=47 x-179$ & $45+/-1$ & 2.89 & 1.9 \\
\hline & PLA & 0 & No & $y=39 x-159$ & $61+/-2$ & 2.85 & 2.7 \\
\hline
\end{tabular}


Photomicrograph of PLA Weibull Fracture Surfaces:

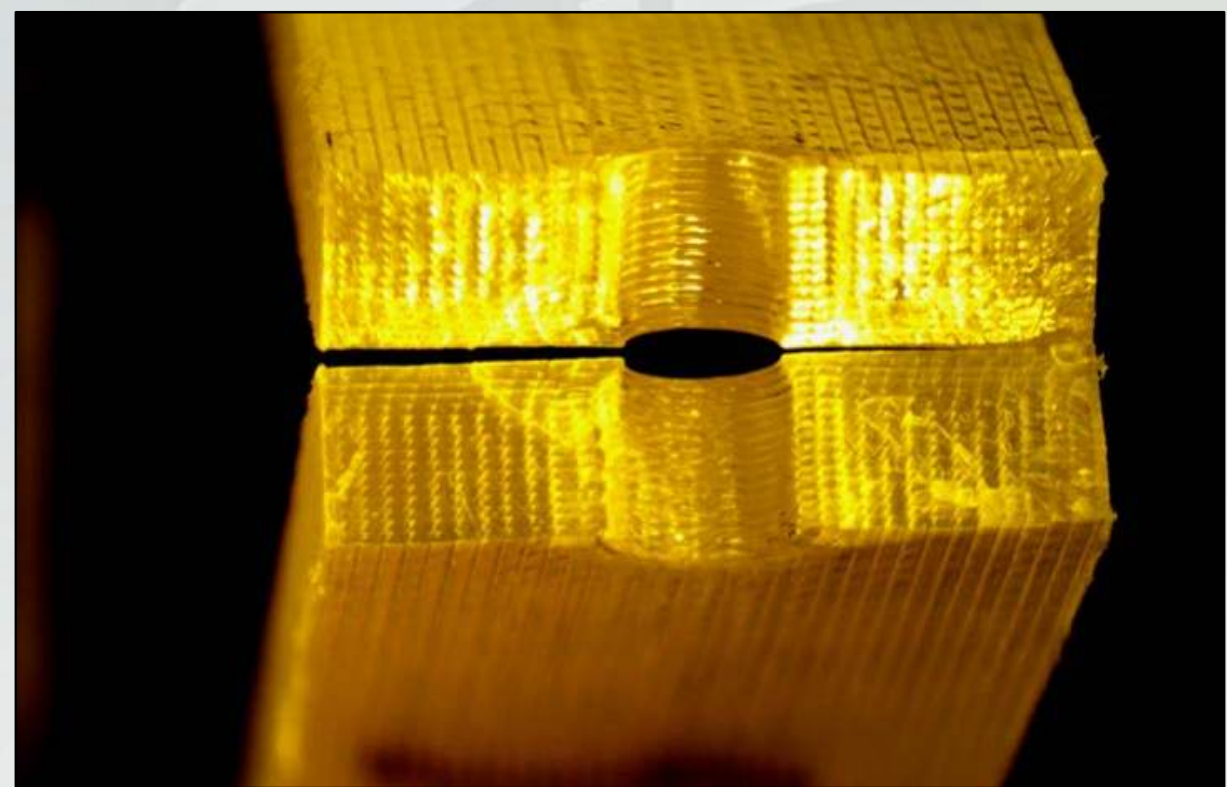




\section{Three distinct groups of Weibull statistical moduli highlighted in Red:}

- Group of low Weibull moduli below $\sim 7$ range in failure across a broad range of stresses, including (from least to greatest) FlashForge ABS $10 \mathrm{wt} . \% \mathrm{M} 50^{\circ}$ raster and Fusion ABS 10 wt.\% $\mathrm{M} 50^{\circ}$ raster.

- Second group has Weibull moduli close to 25 , including Fusion ABS $10 \%$ wt. $\%$ M5 $45^{\circ}$ $45^{\circ}$ raster, Fusion $\mathrm{ABS} 0$ wt. \% M5 $0^{\circ}$ raster, and FlashForge 0 wt. \% M5 $0^{\circ}$ raster.

- Third group has high Weibull moduli in the upper 30s and 40s for PLA samples with and without a hole.

$>$ Overall, high graphene content decreases reliability due to apparent decreased interfilament adhesion, whereas $45^{\circ}-45^{\circ}$ raster increases reliability. 


\section{General Summary:}

- Milled-to-shape fabrication resulted in greater elongations than print-to-shape fabrication due circumferential 3D printing defects.

- Toughness halved with each 5 wt. \% addition of M5 graphene.

- Tensile modulus doubled with 10 wt.\% M5 content.

- Bowden feed resulted in better surface appearance, but poorer filament bead fusion compared to direct feed extrusion.

- Overall, high 10 wt. \% graphene content decreases Weibull reliability due to apparent decreased inter-filament adhesion, whereas $45^{\circ}-45^{\circ}$ raster increases reliability. 


\section{Acknowledgement:}

- Chris Surbrook, Midland Compounding and Consulting

\section{XGSciences}

- Prof. Larry Drzal, P. Askeland - MSU and xGSciences (M5 graphene)

- 3DXTech (Filament Fabrication)

- P. B. Smith - MSU (NMR)

$\underset{\text { Advanced Materials }}{\text { DDXTECH }}$

$\$ !$

- Herbert H. and Grace A. Dow Foundation

- Rollin M. Gerstacker Foundation

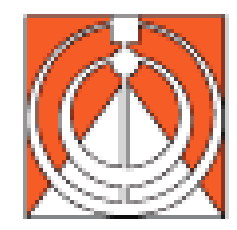

ROLLIN M. GERSTACKER FOUNDATION

- Charles J. Strosacker Foundation

- Dow Chemical Company Foundation

The

C $\overline{\text { HARLES J. STROSACKER }}$ FOUNDATION 


\section{We and our STEM high school students thank}

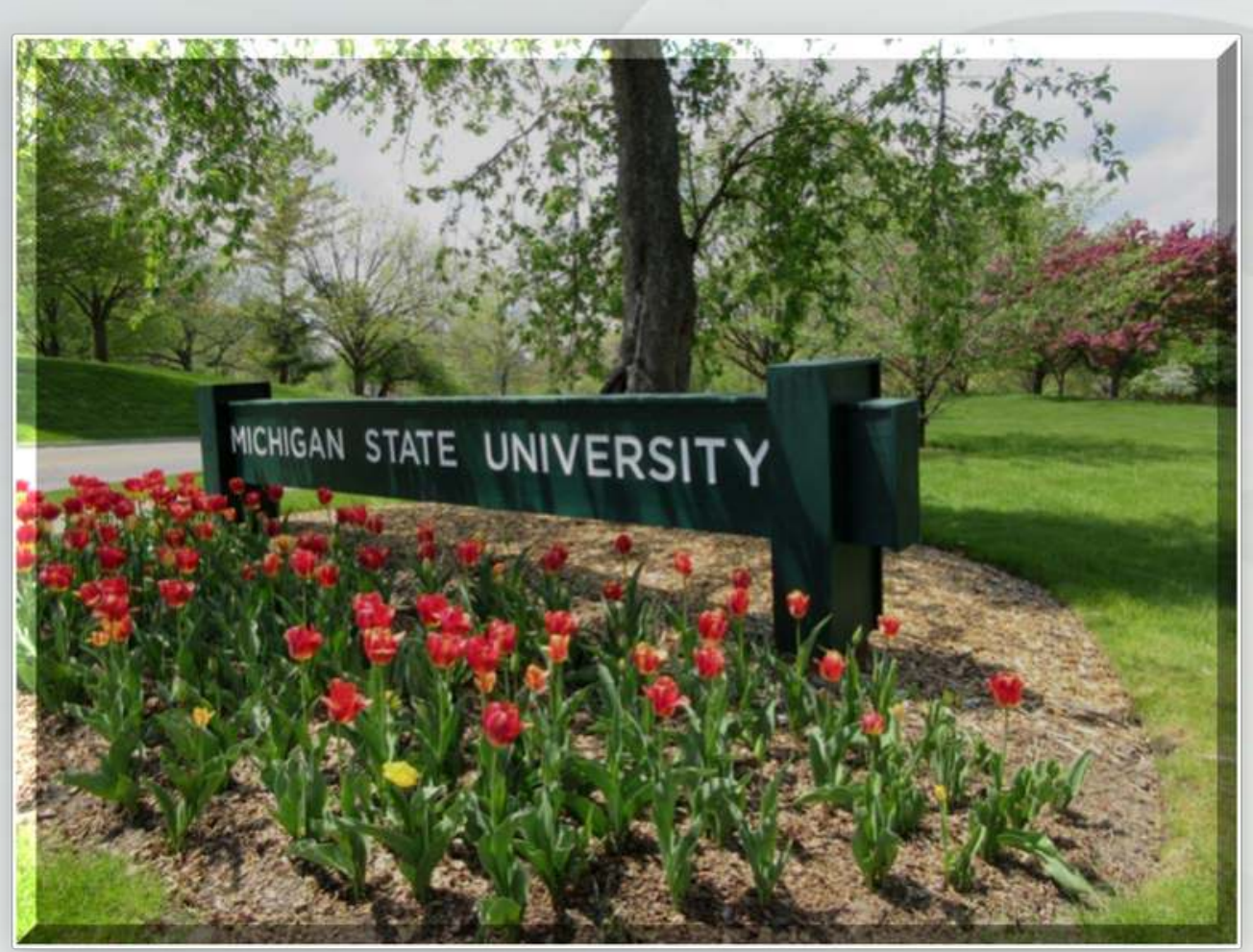

\author{
you for your \\ attention
}

and interest. 